\title{
Research of Polymer Planar Optical Waveguide Sensing Characteristics
}

\author{
Lu Anjiang ${ }^{1,2}$, Bai Zhongchen², Zhang Zhengping ${ }^{1,2}$ and Qin ShuiJie, ${ }^{2, *}$ \\ ${ }^{I}$ College of Big Data and Information Engineering, Guizhou University, Guiyang, Guizhou, 550025, China \\ ${ }^{2}$ Key Laboratory of Optoelectronic Technology and Application of Guizhou Province, Guiyang, 550025, China
}

\begin{abstract}
In order to research the sensing characteristics of polymer optical waveguide. In this paper, the theoretical model of transmission of three-layer polymer planar optical waveguide was established based on coupled-mode theory. The working principle of optical waveguide biosensor was investigated. The article also researched the relationship between an optical guide's physical parameters and surface sensitivity and improved on the sensitivity calculation method of composite optical guide sensing components. This paper then goes on to establish a sensitivity analysis method for disappeared wave type sensing components. Based on this, the article has developed sensitivity calculation software for high sensitivity optical guide sensing components and improved film refraction rate of the method for sensitivity calculation.
\end{abstract}

Keywords: Planar Optical, Polymer, Sensor, Waveguide Bragg Grating.

\section{INTRODUCTION}

Broadband communication can be achieved through free space optics and waveguide optics. Free-space optics (FSO) refers to the modulation of visible light and infrared for broadband communications $[1,2]$. This method can be used within a range of a few kilometers which may be influenced by rain, snow, dust and/or fog. Fiber is divided into optical fiber waveguide and planar waveguides based on waveguide optics theory. Silica fibers are widely used in high-speed data transmission, but it is not conducive to the integration of high densities. Planar light wave circuit devices can diversify its device integration. Planar integrated optics and waveguide are used for short distance optical networks, and also in all-optical networks. Recently, the technology of dense wavelength division multiplexing (DWDM) for optical communication has made a revolutionary progress. DWDM uses multiple wavelengths as a carrier to allow the carrier channels simultaneously in the fiber-optic transmission [3].

Polymers have unique advantages in the fabrication of optoelectronic devices. Preparation technology of polymer optical waveguide devices have more experience and better compatibility in cases where multiple types of substrate, lasers, modulators, detectors, sensors can be integrated into a single chip. Good flexibility of polymers can be used for both vertical and horizontal interconnection devices [4]. The gray-scale gradient of exposure and etching of polymer offers promising prospects for vertical integration and integrated stereo three-dimensional space and flexible polymer optical waveguide devices. Polymers are a very promising development with wide application prospects as the base material. At present, the polymers have been used in the production of arrayed waveguide grating Multiplexer, optical switches, amplifiers, modulators and micro-ring resonators.

*Address correspondence to this author at the Key Laboratory of Optoelectronic Technology and Application of Guizhou Province, Guiyang, 550025, China; E-mail: 39146565@qq.com
Photonics polymer devices have been involved in almost every field. Polymer materials have made a breakthrough in optical loss and optical amplification in recent years [5].

In the Photonics field of high-tech in the world today, sensors manufactured with polymer planar waveguide technology have become the focus of much research. Compared with the traditional use of sensors to detect electrical signals, a polymer planar waveguide sensor uses an optical signal for detection which has the advantages of light quality, antielectromagnetic interference capability, low power consumption, wide working frequency range, and stable performance. Compared with the fiber optic sensors, a polymer planar waveguide sensor has the following advantages: (1) it has a wide choice of materials, flexible structural design and much higher sensitivity. (2) It can achieve multiple channels at the same time and the system functions on a substrate integrated waveguide. (3) It can reduce the producing cost by using the semiconductor technology for mass production. Based on the above advantages, the polymer planar waveguide sensor is very significant and has market prospects and development potential [6-8].

The direction of optoelectronics development is the integration of functions and the amount of integration. Functional integration is used to integrate different functional components into a single chip which is miniaturized with high accuracy and high reliability. It can be combined with beneficial properties of discrete devices and can optimize the overall integrated performance. The amount of integration is designed to include several single devices together in forming arrays of devices, achieving a kind of discrete device that cannot achieve functionality, device miniaturization or integration.

Optical integrated mixed forms can be divided into single-chip integration and mixed integration. Monolithic integration is on the same substrate as production technology and integration technology in integrating all components in one place. Mixed integration is the result of a mixture of 
different production processes after making some components, installation or other optical crystals in semiconductor substrate of the assembly technology.

From the perspective of space, light integration can be divided into two-dimensional and three-dimensional integrations. Two-dimensional integrated development is a synthesis of planar optical waveguide devices. Three-dimensional optical waveguide for integration provides ample space and ideas for the fabrication of optical waveguide devices on a plane and on a different surface and different coupling modes where integrated optical waveguide devices are implemented. Compared to a two-dimensional integration, the three-dimensional integration process has more complexity and difficulty of preparation. Three-dimensional space achieves integration through the two methods of vertical coupling of lithography and gradient etching.

With the further development of optical technology, some problems were discovered in inorganic optical waveguide devices including cost, silicon-based optical switch driving power, wavelength tuning range, etc. So the choice of materials in waveguide devices becomes a focus. In order to get highly-integrated optical components, polymer is considered as an option $[9,10]$. High performance polymer materials for integrated optical waveguide devices are very important because the polymer is simple and it can improve the performance and reduce costs. For example, polymer reduces optical loss in the device. In addition, devices with optical power can zoom or the electro-optical properties of devices can be improved by doping the polymer devices. Because polymer optical waveguide devices have more advantages, so the study of polymer optical waveguide devices is very much needed [11].

\section{OPTICAL WAVEGUIDE PRINCIPLE OF RE- FRACTION}

Waveguides can make waves in transverse or longitudinal directions to be restricted to waves along a certain direction and inhibit dissipation of energy. Waveguides are divided into flat, rectangular, ridge and the loading bar based on waveguide cross-sectional shapes. They are further divided into multimode interference (MMI), long/short periodic grating, arrayed waveguide grating (AWG), Mach-Zander Interferometer (MZI), Directional Coupler (DC) and the microring resonator (MR) and many other types based on waveguide planar structures. Optical waveguide is a basic structure consisting of many optoelectronic devices such as lasers, wavelength division multiplexers, modulators, amplifiers, attenuators, sensors, etc.

The structure of planar optical waveguide devices and design of its scale is necessary to analyze optical waveguide transmission. The effective refractive index of optical waveguides determines the characteristics of light. We can resolve various models of the effective refractive index with the Helmholtz equation and boundary conditions. The equation for non-symmetrical three-layer planar waveguide and guided modes of a rectangular waveguide structure has been derived in this section.

The simplest structure consists of a three-layer dielectric waveguide. In the middle of the dielectric layer is the core called the cladding layer and both sides are called the media. N1, N2, N3, respectively are the refractive index of the waveguide core, lower and upper cladding. Incidence and refraction of beam meet the Snell's law of refraction at the interface.

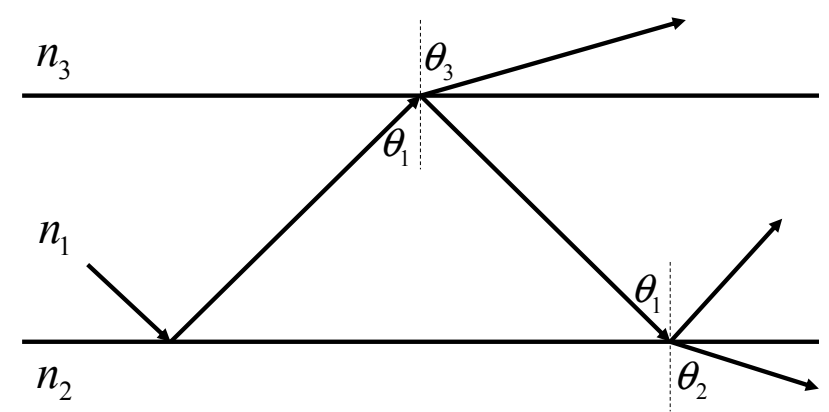

Fig. (1). Reflection and refraction of light.

$n_{1} \sin \theta_{1}=n_{2} \sin \theta_{2}$

$n_{1} \sin \theta_{1}=n_{3} \sin \theta_{3}$

$n_{1} \sin \theta_{1}=n_{2} \sin \theta_{2}=n_{3} \sin \theta_{3}$

There is total reflection for beams in the two boundaries at the same time. There is no refraction of light in the upper and lower layer. Light spreads along the $\mathrm{z}$ direction in a waveguide core layer called the guided mode.

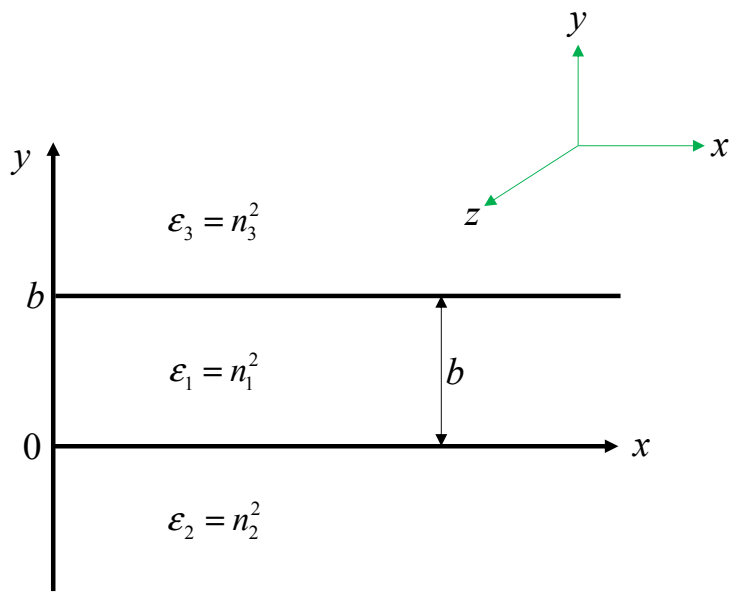

Fig. (2). Three-layer planar waveguide cross section.

There are two basic patterns in a slab waveguide. If we select the electric field along the y-direction which is parallel to the waveguide interface polarization, then the electric field perpendicular to the z-direction is horizontal. This transverse electric mode is abbreviated as TE-mode. If we select the direction of the magnetic field which is parallel to the waveguide interface y polarization, then the magnetic field perpendicular to the z-direction will be horizontal. This transverse magnetic mode is abbreviated as TM-mode.

Combined with Helmholtz equations and boundary conditions, we can obtain the characteristic equation of nonsymmetrical three-layer planar waveguide. 
$\varepsilon(x)=\left\{\begin{array}{cc}\varepsilon_{2} & (-\infty<\mathrm{x}<0) \\ \varepsilon_{1} & (0 \leq \mathrm{x} \leq \mathrm{b}) \\ \varepsilon_{3} & (\mathrm{~b}<\mathrm{x}<\infty)\end{array}\right.$

$\gamma_{1} b=m \pi+\arctan T_{2}+\arctan T_{1}$

When $\mathrm{S}=0$, it is in the TE-mode. When $\mathrm{S}=1$, it is in TMmode.transverse electric mode (TE-mode). If we select the direction of magnetic field which is parallel to the wave guide interface y polarization and magnetic field perpendicular to the z-direction is horizontal, this model is called as transverse magnetic mode (TM-mode).

Combined with Helmholtz equations and boundary conditions we can obtained the characteristic equation of nonsymmetrical three-layer planar waveguide.

$\varepsilon(x)=\left\{\begin{array}{cc}\varepsilon_{2} & (-\infty<\mathrm{x}<0) \\ \varepsilon_{1} & (0 \leq \mathrm{x} \leq \mathrm{b}) \\ \varepsilon_{3} & (\mathrm{~b}<\mathrm{x}<\infty)\end{array}\right.$

$\gamma_{1} b=m \pi+\arctan T_{2}+\arctan T_{1}$

When $\mathrm{S}=0$, it is for the TE mode. While $\mathrm{s}=1$, it is for TM mode.

\section{CHARACTERISTICS OF OPTICAL WAVEGUIDE SENSORS}

In this type of sensor, a sensitive reagent fixed on the surface of optical waveguide film affects the object and changes the waveguide propagation mode of light which is usually seen on the propagation of light in the case of loss or displacement. These factors change the output light intensity. Output light intensity levels associated with the amount of output light intensity detection can be obtained by measuring the concentration of information.

Compared with other chemical sensors, optical waveguide sensors have the following characteristics:

(1) The optical waveguide is very sensitive to ambient refractive index changes in the waveguide layer. Optical chemical sensor sensitivity is good.

(2) A high sensitivity can be achieved through keeping the sensitive material and waveguide material and structure optimization design.

(3) It has high mechanical strength and resistance to electromagnetic interference. These sensors are small in size and can work at room temperature.

(4) Single-chip integration can achieve multiple channels at the same time and system miniaturization and multiplefunctions, as well as reducing power consumption.

(5) It can provide new sensing applications and integration potential by combining with micromachining and microelectronics technology.
(6) It can reduce costs through an integrated process to achieve mass production.

Based on evanescent wave sensors of the optical waveguide field strength enhancement mechanism, the current optical waveguide surface Plasmon resonance chemical sensors can be divided into surface Plasmon resonance (SPR) and flat glass optical waveguide sensors.

\subsection{Surface Plasmon Resonance}

The physical optics phenomena of Surface Plasmon resonance was found in optical waveguide. Due to special optical properties of metals, metal at the interface of surface Plasmon resonance waves can be transmitted. A beam of incident plane is monochromatic when polarized light at an angle to the glass surface coated with a thin layer of gold film, and total internal reflection occurs. If the wave vector of the incident light and the surface electron oscillation frequency of occurrence within the gold film, caused resonant light is coupled into the gold film which is called as surface Plasmon resonance. Surface Plasmon resonance of metal near the surface of the refractive index is extremely sensitive to any change. The researchers used this characteristic to develop the waveguide surface Plasmon resonance chemical sensor. The sensor structure chart is shown in Fig. (3).

In the modern development of science and technology, both humans and machines play a role. People rely on the development of information resources in modern society. Information resources transmit and process with sensors in research objects and control systems of interface locations in industrial production, such as for scientific experiments, and other activities. Detection information of a sensor window is all about perception, and it provides a system for decisionmaking and processing of original data. The sensor ascertains the nature of tentacles and its role is similar to the human senses. If the computer is equivalent to the human brain, the executor is equivalent to the human body and the sensor is equivalent to human facial features. If a person's facial features are not accurately expressing the true feelings of that person, the mind of the observer will reach the wrong conclusions and will behave in an erratic and chaotic manner. This shows the importance of sensors. Sensors have sensing functions similar to those of humans. However, human senses cannot perceive important information beyond human reach and are unable to endure harsh environments which are an indispensable component of modern information measurement and control systems.

All scientific and production processes obtain information with an automatic detection and control system. Sensors are required for easy transmission and processing of the signals. If there are no sensors for accurate and reliable measurements of the original parameters, or if the sensor is not sensitive to feelings or measurement, it cannot be converted to electrical signals which are measured accurately, thus rendering other instruments and apparatuses of high precision useless. 


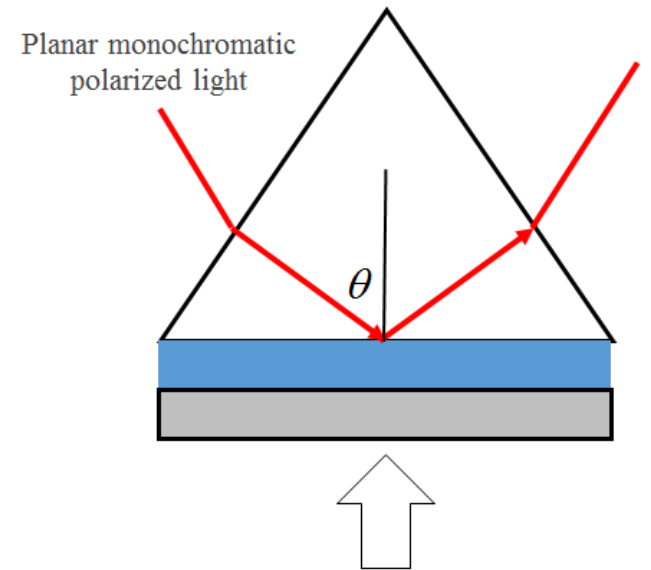

The measured object

Fig. (3). Structure of SPR sensor.

\subsection{Flat Glass Optical Waveguide Sensors}

Some compounds for sensitive material and film are selected and fixed onto the surface of the $\mathrm{k}+$ ion-exchanged glass waveguides. The prism coupling method is used to insert a laser beam into the optical waveguide. When it enters at the same angle as the special angle of incidence of the light, light is constrained to the guided wave level of total internal reflection and transmission. Flat-glass optical waveguide sensors have high sensitivity and rapid response, pertaining to the simple fabrication process, etc. The sensitivity of Planar waveguides is related to the waveguide refractive index, the thickness of the layer, the sensitive layer, the sensitive layers of light absorption and the scattering factors. Therefore, a chemical sensor with high sensitivity and selectivity can be obtained by choosing the guiding layer and sensitivity with flexibility.

$$
\begin{aligned}
& k_{0} Z_{c}=\frac{\tan \theta}{\sqrt{\left(N^{2}-n_{c}^{2}\right.}} \\
& X_{c}=\frac{Z_{c}}{\tan \theta}=\frac{1}{k_{0} \sqrt{\left(\mathrm{N}^{2}-\mathrm{n}_{c}^{2}\right)}}
\end{aligned}
$$

The principle of an optical chemical sensor is based on evanescent waves. Evanescent waves are inherent characteristics of dielectric optical waveguide which has no special requirements for the waveguide material. It has the advantages of high sensitivity and is easily implemented.

The total internal reflection of light occurs in the guiding layer and forms the guided mode. Light waves are not at all reflecting the guiding layer at the interface. Instead, through interfaces, they must penetrate the cladding and base plate height, and through displacement, return the guiding layer. The light waves that penetrate the cladding and base plate are called evanescent waves and their electric field intensity decreased according to an exponential decay.

$$
\begin{aligned}
& E_{i}=A_{i} \exp \left(-x / X_{i}\right) \\
& X_{i}=\frac{\lambda}{2 \pi \sqrt{N^{2}-n_{i}^{2}}}
\end{aligned}
$$

\section{RESEARCH ON SENSING PROPERTIES}

\subsection{Temperature Sensors}

Polymer planar waveguide temperature sensors use a planar light wave circuit as the carrier and it can sense changes in temperature and converts the optical output signal to a new generation of sensors. Compared to other types of temperature sensors, polymer planar waveguide temperature sensors include the advantages of anti-electromagnetic interference capability, a wide working frequency range, a multifunctional single-chip, and low cost. The Polymer waveguide of Bragg grating temperature sensor is a new type of waveguide based on temperature change of the refractive index grating period and Bragg grating wavelength integrated optical temperature sensing elements.

The light enters into the device through a wide spectrum of light output coupling and enters into the spectrometer through the device of coupled light, which uses computers,

\begin{tabular}{|c|c|c|c|}
\hline $\mathbf{T}$ & $\lambda$ & BW $_{3-\mathrm{dB}}$ & $E R / d B$ \\
\hline 16.1 & 1552.4 & 2.3 & 12.1 \\
\hline 26.5 & 1554 & 2.5 & 11.7 \\
\hline 37.3 & 1556 & 2.2 & 12.1 \\
\hline 47.2 & 1558.2 & 2.5 & 11.7 \\
\hline 55.6 & 1560.6 & 2.2 & 12.3 \\
\hline
\end{tabular}
monitors, and cameras, microscopes, display systems to aid in testing, and precision micro bench system for debugging.

Table 1. Bragg gratings at different temperatures.

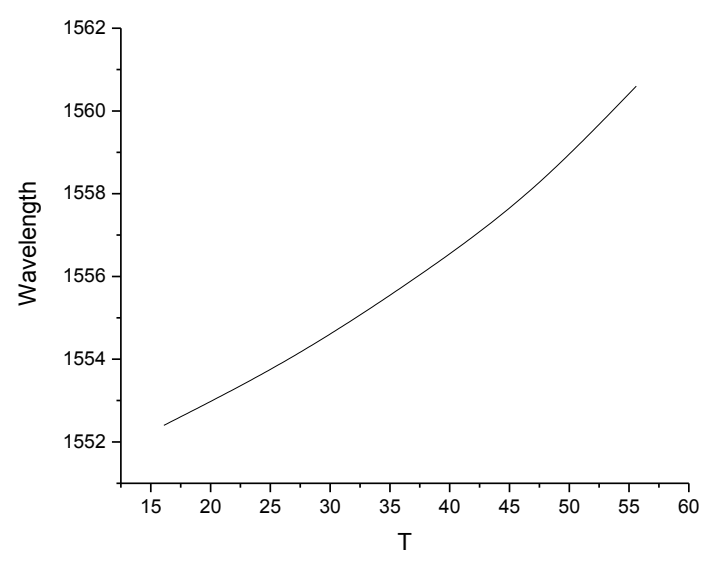

Fig. (4). Bottom structure of corrugated waveguide bragg grating resonant wavelength changes in temperature.

\subsection{Refractive Index Sensors}

The refractive index is a basic parameter of liquid. In daily life, a liquid refractive index is required to obtain its parameters such as temperature, concentration and density. There is a need for real time detection of concentration and 
density in the area of food production, medicine and health. So accurate detection of liquid refractive index is of great value in many fields.

$$
\begin{aligned}
& \Delta \phi=2 \pi L\left(N_{1}-N_{2}\right) / \lambda_{0} \\
& P_{\text {out }} / p_{\text {in }}=\cos ^{2}(\Delta \phi / 2)
\end{aligned}
$$

The equation shows that MZI waveguide output light intensity depends on the difference in the transmission phase of two branches of arms. We can carve eclipse on one of the support waveguide package layers and make it as a sensing arm and the other arm as a reference arm. We make transmission light of evanescent field energy as a distribution in outside air in the dang to sense arm drops and liquid. Due to the evanescent field's interference, transmission light of energy distribution will occur, and will lead to changes in the sensing arm waveguide of guide mode effective refraction rates. The two arms of transmission phase with change cause strong changes in output light.

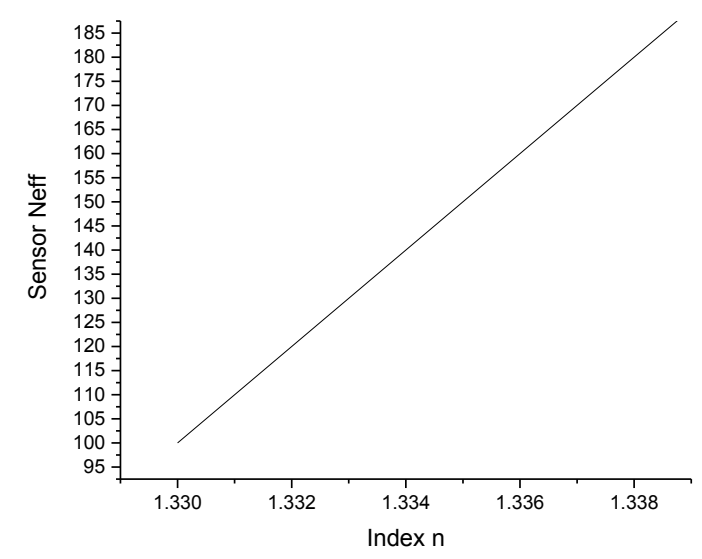

Fig. (5). Refractive index sensing arm for measuring changes in liquid refractive index.

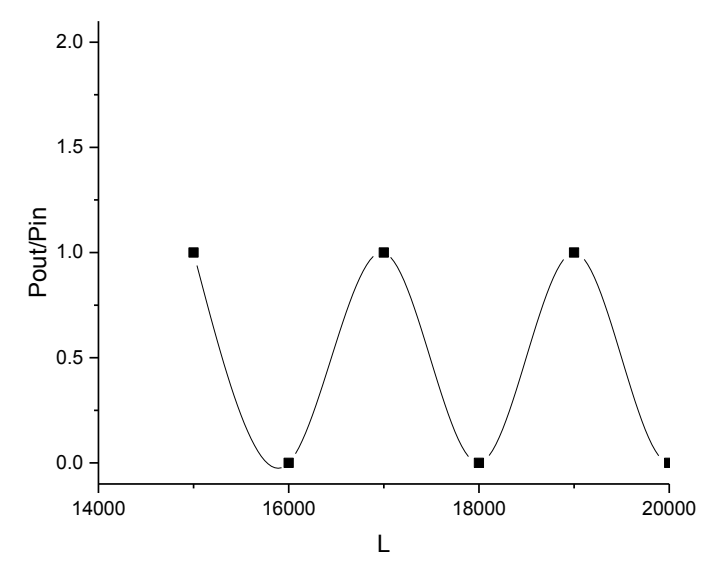

Fig. (6). Normalized output changes with sensor window.

\section{CONCLUSION}

At present, the information age is developing. Optical communications is an important technology building up the information society. Therefore, integrated optics has become a field of focus for international scholars. Polymer planar optical waveguide is a significant research topic in the field of integrated optics. The polymer planar optical waveguide used as a sensor is a new attempt which is very helpful to the future development of sensors.

Preparation of optical waveguide sensor surface sensitivity calculation by sensitive components through structural adjustment is crucial. The optical properties of the electromagnetic theory of light and medium based on calculation method of relative sensitivity of the optical waveguide sensors was optimized in the research of this paper. Results show the relative sensitivity of optical waveguide sensors and surface film of refractive index and thickness, as well as guided-wave optical wavelength. Results of the study on the preparation of sensor laser light source selection provide a theoretical foundation. Programming with VB.NET language sensitivity has been developed on the basis of this calculation and the drawing software which is easily calculated for data analysis. The improved calculation of the refractive index method for sensitivities calculation provides a reliable parameter.

This thesis uses electronic communication and intelligent design of instrument automation to test hardware architecture. The exposure meter is connected to the computer with a data acquisition card and using VB.NET programming language. The supporting optical waveguide testing system has been developed for data collection and analysis software. The detection system connects to a computer so as to solve the problem of the original system which cannot detect data, as well as the problem of processing and storing information being inconvenient. The application software does not need any software support, and has a user-friendly interface, is easy to operate and has high operational stability. We can use the newly designed optical waveguide testing system based on optical waveguide with high sensitivity detection of sulfur dioxide and hydrogen sulfide gas sensor. We can use the new design of optical waveguide testing system, for which the research team has developed a detection method for acid gases and volatile organic compounds in chemical sensors of optical waveguide sensors with high sensitivity. We proved that the new design's detection system is more powerful than the original detection system. It is accurate, sensitive and bringing great convenience to this kind of detection.

This thesis is about the fundamental research of the polymer MZI waveguide liquid refractive index sensor and the polymer waveguide Bragg grating temperature sensor. The main findings are: (1) In the polymer MZI waveguide liquid refractive index sensor, the length of sensing window was optimized by means of the $\mathrm{VC}++$ software programming. In the chosen measuring range, the monotonicity change in the measurement curve can be realized, and the higher sensing sensitivity can be obtained. The device which had very good performance was fabricated taking advantage of microelectronics technology, and the stability of the test system is improved by the aluminum probe device fixed on the test plat- 
form. The refractive index sensing function is realized by testing the relationship between the output optical power and the measured liquid concentration. (2) In the polymer waveguide Bragg grating temperature sensor, according to the existing process conditions, the grating structure is reasonably designed. The grating characteristic parameters are analyzed by means of the MATLAB software. The Bragg waveguide grating filter, which has good performance, is prepared by using ultraviolet lithography and ICP etching. The temperature sensing function is realized by testing the drift of the resonance wavelength with the temperature change, with a sensor sensitivity of $0.20686 \mathrm{~nm} /{ }^{\circ} \mathrm{C}$.

\section{CONFLICT OF INTEREST}

The authors confirm that this article content has no conflict of interest.

\section{ACKNOWLEDGEMENTS}

This work is supported by the National Natural Science Foundation of China (Grant No.11204046), the International S\&T Cooperation Projects of China (2014DFA00670), and the social development projects of Guizhou province (Guizhou SY [2013]3105).

\section{REFERENCES}

[1] SW James, ML Dockney, and RP Tatam. Simultaneous independent temperature and strain measurement using in-fibre Bragg grating sensors. Electronics Letters 1996; 32(12): 1133-4.
[2] Woong-Gyu Jung, et al. High-sensitivity temperature sensor using a side-polished single-mode fiber covered with the polymer planar waveguide. Photonics Technology Letters, IEEE 2001; 13(11): 1209-11.

[3] M Hong, A K-Y J, and LR Dalton. Polymer-based optical waveguides: materials, processing, and devices. Advanced materials 2002; 14(19): 1339-65.

[4] MBJ Diemeer, et al. Polymeric phased array wavelength multiplexer operating around $1550 \mathrm{~nm}$. Electronics letters 1996; 32(12): 1132-3.

[5] Noh, Young-Ouk, et al. Continuously tunable compact lasers based on thermo-optic polymer waveguides with Bragg gratings. Optics Express 16.22 (2008): 18194-18201.

[6] Chen, Ray T., et al. Guided-wave planar optical interconnects using highly multiplexed polymer waveguide holograms. Lightwave Technology, Journal of 10.7 (1992): 888-897.

[7] K Tiefenthaler, W Lukosz. Optical sensor for selective detection of substances and/or for the detection of refractive index changes in gaseous, liquid, solid and porous samples: U.S. Patent 4,815,843[P]. 1989-3-28.

[8] T Pustelny. et al. Impressing technology of optical Bragg's gratings on planar optical sol-gel waveguides. Opto-Electronics Review 2006; 14(2): 155-160.

[9] M Rosenberger, et al. Compressive and tensile strain sensing using a polymer planar Bragg grating. Optics express 2014; 22(5): 548390 .

[10] M Rosenberger. et al. TOPAS based humidity insensitive polymer planar Bragg gratings for temperature and multi-axial strain sensing. International Seminar on Photonics, Optics, and Applications 2014. International Society for Optics and Photonics, 2015.

[11] James C Gates, et al. UV Written Planar Bragg Grating Sensors-An Overview of Fabrication, Geometries and Applications. Optical Sensors. Optical Society of America, 2013.

Received: February 17, 2015

Revised: May 21, 2015

Accepted: June 09, 2015

(C) Anjiang et al.; Licensee Bentham Open.

This is an open access articles licensed under the terms of the Creative Commons Attribution-Non-Commercial 4.0 International Public License (CC BY-NC 4.0) (https://creativecommons.org/licenses/by-nc/4.0/legalcode), which permits unrestricted, non-commercial use, distribution and reproduction in any medium, provided that the work is properly cited. 